\title{
ANTARCTIC WAVE DYNAMICS MYSTERY DISCOVERED BY LIDAR, RADAR AND IMAGER Cao Chen ${ }^{1 *}$, Xinzhao Chu ${ }^{1}$, Weichun Fong ${ }^{1}$, Xian Lu ${ }^{1}$, Adrian J. McDonald ${ }^{2}$, Dominique Pautet ${ }^{3}$, and Mike Taylor ${ }^{3}$

\author{
${ }^{1}$ University of Colorado at Boulder, 216 UCB, CIRES, Boulder, CO 80309, USA, Email: Cao.Chen@Colorado.edu \\ ${ }^{2}$ University of Canterbury, Department of Physics and Astronomy, Christchurch, NZ \\ ${ }^{3}$ Center for Atmospheric and Space Sciences, Utah State University, Logan, UT, USA
}

\begin{abstract}
Since the start of the McMurdo Fe lidar campaign, largeamplitude $(\sim \pm 30 \mathrm{~K})$, long-period ( 4 to $9 \mathrm{~h}$ ) waves with upward energy propagating signatures are frequently observed in the MLT temperatures. Despite its frequent appearance, such type of wave was neither widely observed nor well understood in the past. At McMurdo $\left(77.8^{\circ} \mathrm{S}, 166.7^{\circ} \mathrm{E}\right)$, the simultaneous observations of such waves using lidar, radar and airglow imager can provide 3-D intrinsic wave-propagation properties, which are greatly needed for understanding their sources and potential impacts. This study presents the first coincident observation of these 4-9 $\mathrm{h}$ waves by lidar, radar and airglow imager in the Antarctic mesopause region.
\end{abstract}

\section{INTRODUCTION}

Although observations in the Antarctic middle and upper atmosphere started very early, there were no rangeresolved temperature measurements at McMurdo until the start of Chu group's lidar campaign [1]. Since then, many new discoveries have been made by lidar observations in Antarctica, e.g., the thermospheric neutral iron (Fe) layers [1], solar effects on Fe layer bottomside, two simultaneous Inertia Gravity Waves (IGWs) [2], large eastward planetary waves and super-exponential growth of thermal tide amplitude above $100 \mathrm{~km}$. These discoveries are only made possible by the robustness and powerfulness of the lidar system. To achieve this, Chu group members have upgraded the lidar several times, and have been constantly maintaining the system well. Therefore, this lidar is capable of running continuously for several days with high performance, which enables the statistical study of wave occurrence and properties.

Benefited from the capabilities of the Fe Boltzmann lidar, a completely new wave phenomenon has been discovered at McMurdo, i.e., strong and persistent 4 to $9 \mathrm{~h}$ period waves with short vertical wavelengths $\left(\lambda_{z}\right)$ from the stratosphere all the way to the lower thermosphere that occur all year round, e.g., [1,2]. However, after its discovery, the true identity of these waves still remains a mystery to the community: Are these waves ordinary IGWs, or short-period Atmospheric Normal Modes (ANMs), or something unknown before? Initial case studies have suggested that some of these waves are ordinary IGW [2]. However, persistent appearance of these waves over McMurdo contradicts the current understanding of Gravity Waves (GWs) being intermittent. Furthermore, what could be the persistent sources for these waves around Antarctica? There are no commonly known sources, especially in summertime when polar vortex has disappeared. On the other hand, ANMs, natural resonant modes of atmospheric free oscillations [3] or $8-\mathrm{h} / 6-\mathrm{h}$ tides can be possible causes. However, these explanations cannot solve the mystery because the observed vertical wavelength $\lambda_{z}(\sim 20 \mathrm{~km})$ are much shorter than those predicted for ANMs or tides according to both theory and simulations. The lack of a convincing theory for these 4-9 h waves in Antarctica has put forth a big mystery.

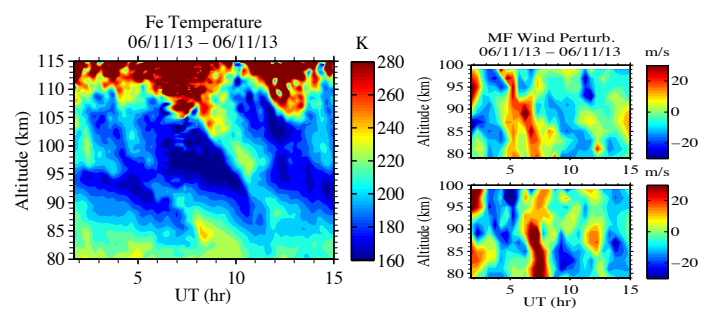

Figure 1. (a) Raw temperatures from 1.5 to 15 UT on 11 June 2013 by lidar. (b) Zonal wind (top) and meridional wind (bottom) data by MF radar at the same time with the 12-h and 24-h tides removed.

Solving this mystery is crucial to improving the current weather and climate models and to understanding climate changes especially in Polar Regions. Currently, models have simulated large-scale waves such as planetary waves and tides well, but most of them cannot directly resolve small-scale waves such as GWs and ANMs. These small-scaled waves, although smaller in scales, transport significant amounts of energy and momentum up to the MLT region where they strongly influence the mean wind and alter the temperature structure. Therefore, this incapability has become the biggest uncertainty in weather and climate forecasting models and has greatly undermined the models' predictive capability.

To resolve this mystery, we need the 3-dimensional (3-D) intrinsic wave properties, i.e., observed and intrinsic periods, horizontal propagation direction, wavelengths $\lambda_{h}$ and $\lambda_{z}$. Fortunately, two other instruments collocated with our Fe Boltzmann lidar enable such study. One is Scott Base MF radar, which has continuous temporal coverage and measures MLT winds between 70 and $100 \mathrm{~km}$. 
Combining our lidar with this radar, intrinsic wave properties can be derived, e.g., [2]. Another is Utah State University (USU) all-sky infrared imager provides measurements on the intensity of infrared $\mathrm{OH}$ emission layer $(\sim 87 \mathrm{~km})$. The $\mathrm{OH}$ imager data can provide horizontal propagation information directly.

\section{CASE STUDY}

\subsection{Simultaneous and common volume observations of the 4-9 $\mathrm{h}$ waves by lidar, radar and imager}

On 11 June 2013, strong wave perturbations with period $\tau$ $\sim 5 \mathrm{~h}$ were observed in Fe lidar temperatures as shown in Figure 1a. This wave has downward phase progression and $\lambda_{z}$ of $\sim 20 \mathrm{~km}$. The raw MLT temperature data have resolutions of $0.1 \mathrm{~h}$ and $0.1 \mathrm{~km}$. The data we used were smoothed temporally and vertically with Hamming windows of $0.5 \mathrm{~h}$ and $1 \mathrm{~km}$ FWHM. Therefore, only the waves with $\tau \geq 1 \mathrm{~h}$, and $\lambda_{z} \geq 2 \mathrm{~km}$ were resolved. MF wind data also shows a 5 -h wave as in Figure $1 \mathrm{~b}$, after removing the semidiurnal and diurnal tides, we can see large wind perturbations in both the zonal and meridional directions. We first assume this wave as IGW, and then test it with a linear GW theory. At the same time, the 5-h wave was clearly observed by the $\mathrm{OH}$ imager. Figure 2 shows two keograms, one for the South-North (S-N) direction and another for West-East (W-E), which are composed of the S-N and W-E slices at the center of each raw image proceeding with time. The horizontal ranges over the S-N and W-E directions are 280 and $350 \mathrm{~km}$, while the temporal and spatial resolutions are $0.5 \mathrm{~min}$ and $\sim 1 \mathrm{~km}$, respectively.

\section{Data Analysis}

In order to minimize contamination of tidal and planetary wave oscillations, the temperatures, winds and $\mathrm{OH}$ intensities are all band-pass filtered by a 6th-order Butterworth filter with pass band between $2.5-7 \mathrm{~h}$. We then fit two $\mathrm{OH}$ intensity keograms to a non-linear monochromatic wave model to extract the horizontal propagation properties of the waves. The model $I(t, x, y)$ which takes two forms for each keogram, is written explicitly as follows.

$$
\begin{aligned}
& I(t, x, 0)=A I_{x} \cos \left(2 \pi \omega t-2 \pi k x+\phi_{0}\right)+I_{x 0} \\
& I(t, 0, y)=A I_{y} \cos \left(2 \pi \omega t-2 \pi l y+\phi_{0}\right)+I_{y 0}
\end{aligned}
$$

In this model, $\omega$ is observed frequency, $k$ is the zonal wavenumber, and $l$ is meridional wavenumber. The fitting process is to minimize the chi-square fitting error.

$\chi^{2}=\sum_{i} \frac{\left(I\left(t_{i}, x_{i}, 0\right)-\Upsilon\left(t_{i}, x_{i}, 0\right)\right)^{2}}{u I\left(t_{i}, x_{i}, 0\right)^{2}}+\sum_{j} \frac{\left(I\left(t_{j}, 0, y_{j}\right)-\Upsilon\left(t_{j}, 0, y_{j}\right)\right)^{2}}{u I\left(t_{j}, 0, y_{j}\right)^{2}}$

We utilize a Monte-Carlo sampling method to do the fitting [4]. Basically, for each step of the fitting, a set of random parameters around the current model parameters was generated. Then, a new $\chi^{2}$ is calculated. If the new $\chi^{2}$ is smaller than the pervious one, then this new set of model parameters was accepted. We continue this search until the fitting error is below our tolerance, and the parameters are converged. This method avoids the entrapment in local likelihood maxima, and therefore a useful way to solve non-linear fitting problems. $\lambda_{h}$ and propagation direction are then derived:

$$
\begin{aligned}
& \lambda_{h}=\frac{1}{\sqrt{k^{2}+l^{2}}} \\
& \theta=\tan ^{-1}\left(\frac{l}{k}\right)
\end{aligned}
$$

The fitted results are $\lambda_{h} \sim 1760 \mathrm{~km}, \theta \sim 180^{\circ}$.

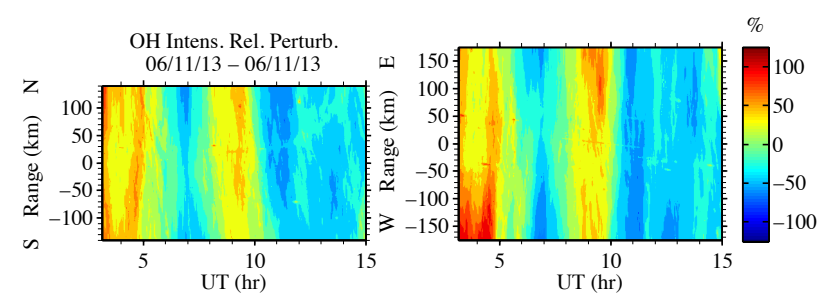

Figure 2. Perturbations of OH intensities on 11 June 2013.

As shown in [2], combining lidar temperatures and radar winds, we can calculate the intrinsic wave properties for IGW. The radar wind vector at each altitude can tell the wave propagation direction but with a $180^{\circ}$ ambiguity. Then by comparing the phase difference between the wind and temperature perturbations, we can resolve this ambiguity. We take a new approach to obtain this phase difference, which is to fit the temperatures and winds together using the following wave model, see [5].

$$
T(t)=|T| \cos \left(2 \pi \omega\left(t-t_{0}\right)\right)+T_{0}
$$

$U(t)=A \cos \varphi \cos \left(2 \pi \omega\left(t-t_{1}\right)\right)-B \sin \varphi \sin \left(2 \pi \omega\left(t-t_{1}\right)\right)+U_{0}$

$V(t)=A \sin \varphi \cos \left(2 \pi \omega\left(t-t_{1}\right)\right)+B \cos \varphi \sin \left(2 \pi \omega\left(t-t_{1}\right)\right)+V_{0}$

where, $t_{0}$ is the phase of temperature perturbation, and $t_{1}$ is the phase of the horizontal parallel wind perturbation. We find the phase difference is $\sim 297^{\circ}$, which is very close to that predicted by linear theory for ordinary IGW, $\left(\sim 270^{\circ}\right)$. This means that our assumption of this wave being ordinary IGW is valid. After the $180^{\circ}$ ambiguity was resolved, we can then use the method in [2] to solve the intrinsic properties. The results are shown in Figure 3.
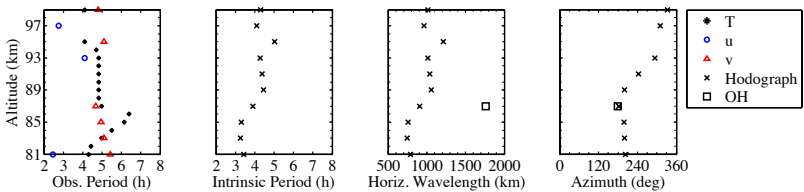

Figure 3. Intrinsic properties calculated from $\mathrm{OH}$ imager keograms and lidar/radar data.

As noticed, the directly fitted $\mathrm{OH}$ keograms yields much longer $\lambda_{h}$ than that calculated from lidar and radar. The discrepancy could be due to the fact that $\mathrm{OH}$ density variations do not have the same response as the temperature variations, due to the chemistry involved. 


\section{STATISTICS}

Although we have reported several case studies of these 4-9 $\mathrm{h}$ waves identified with IGWs [2,6], we believe a statistical study of the wave properties will be more helpful to obtain a comprehensive interpretation of the waves. In this section, we utilize the winter (May, June, and July) temperature data from 2011 to 2014, to derive several statistical wave properties of these 4-9 h waves. We select observation episodes longer than $12 \mathrm{~h}$ to do the study because this allows us to accurately determine the wave periods. A total of 135, 267, 172 hours of data were used in this study for May, June and July, respectively. Figure 4a shows an example of the persistence of these 4-9 h waves in our observations. During nearly 50 hours of observations on 18 June 2014, clear downward phase progressions are always presented. Figure $4 \mathrm{~b}$ shows the corresponding frequency spectra at $85 \mathrm{~km}, 90 \mathrm{~km}$ and 95 $\mathrm{km}$ in which the waves peak at $\sim 5.5 \mathrm{~h}, \sim 6.5 \mathrm{~h}$, and $8 \mathrm{~h}$. Persistent presence and discrete distribution of the dominant periods of the $4-9 \mathrm{~h}$ waves as shown in Figure 4 are very common in our observations.
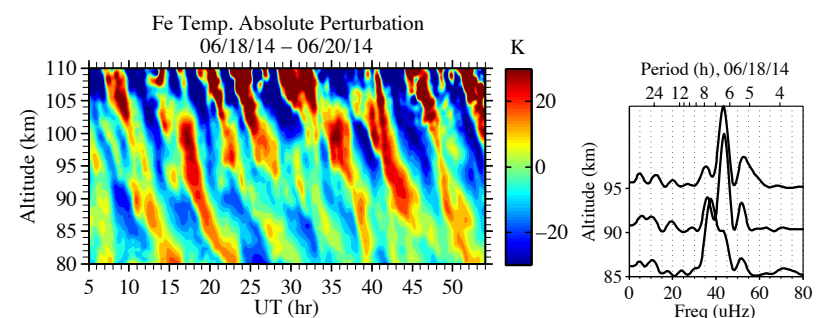

Figure 4. (a) Continuous 50-h temperature measurements show persistent 4-9 h waves on 18-20 June 2014. (b) The frequency spectra at $85 \mathrm{~km}, 90 \mathrm{~km}$ and $95 \mathrm{~km}$.

\section{Wave Frequency Spectra}

Observations have shown that ordinary GWs tend to have a so-called "canonical" wave spectrum, i.e., in the spectral region where waves are regarded as being saturated; the power spectral density (PSD) follows a form of $m^{-p}$ or $\omega^{-q}$ ( $m$ and $\omega$ are the vertical wavenumber and frequency, respectively; $-p$ and $-q$ are the corresponding slope of the power) [7]. According to diffusive filtering theory [7], $p=2 q-1$. The studies done by $L u$ et al. [8] showed that, $-p$ is around -2.26 at McMurdo, therefore, $-q$ should be around -1.6 .
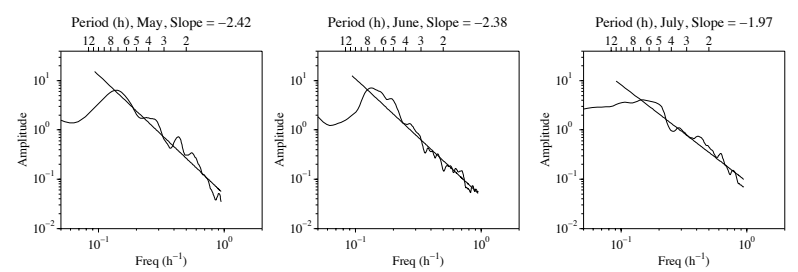

Figure 5. Mean frequency spectra in the MLT for May, June and July. Black dashed lines are fittings of the power law shapes in the range of $\sim 1-11 \mathrm{~h}$. The data used to calculate the spectra have $0.5 \mathrm{~h}$ and $1 \mathrm{~km}$ intervals in time and space.
We plot the mean frequency spectra for each month in Figure 5. Two things are different from the predicted universal power law. First, the slopes in all three months are much steeper than -1.6 . Second, the spectral energies in periods range 4-9 $\mathrm{h}$ are higher than the power law fitting suggesting that these waves do not follow power law and therefore are different from the ordinary GWs.

\section{Occurrence frequency}

Ordinary GWs are believed to be intermittent, and therefore the occurrence frequency of these waves is critical when identifying whether they are ordinary GWs. Because multiple waves were often present at the same time, wavelet techniques were used in analyzing temperature time series at each altitude as described in [9]. Multiple peaks were identified in wavelet power as a function of periods and time, each of which is considered as one wave event. Then the time span of each wave event is determined from the full-width-half-maximum (FWHM) of the corresponding wavelet power peak. Usually, 2-6 wave events can be identified at each altitude during each episode of observation using this method. Figure 6 shows the percentage of the time span of a wave event over entire observation time as a function of the wave period in May, June, July averaged over all altitudes through 4 years of lidar campaign.
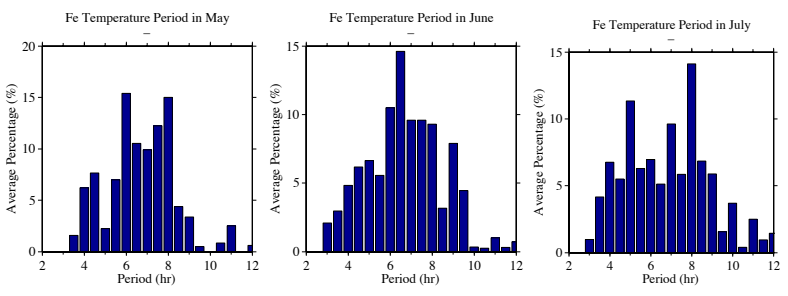

Figure 6. Percentage of the time span of a wave event over entire observation time as a function of the wave period in May, June, July through 4 years of lidar campaign. Results are averaged between 81 and $105 \mathrm{~km}$.

Clearly shown in this Figure 6 is the prevalence of 4-9 $\mathrm{h}$ waves, which are detected by the algorithm $\sim 92 \%, \sim 87 \%$ and $\sim 84 \%$ of the entire observation time in May, June, and July respectively. This high occurrence frequency contradicts the current understanding of GWs being intermittent. Another distinct feature is that the discrete distribution of the dominant periods. For example, in July, the occurrence frequency of waves with $\tau \sim 8 \mathrm{~h}$ is two-thirds higher than the waves in the neighboring period bins. If these are ordinary GWs, this means that the sources of these waves have preference over certain periodicity.

\section{Dominant vertical wavelengths}

To determine the $\lambda_{z}$ of these waves, we first filter the wave perturbations with a 6 -order Butterworth band-pass filter (pass bands between 3 to $12 \mathrm{~h}$ ), and then calculate vertical spatial spectra for each episode, followed by 
averaging over each month. Figure 7 shows the mean vertical spatial spectra. The dominant $\lambda_{\mathrm{z}} \sim 22 \mathrm{~km}$ for all three months.
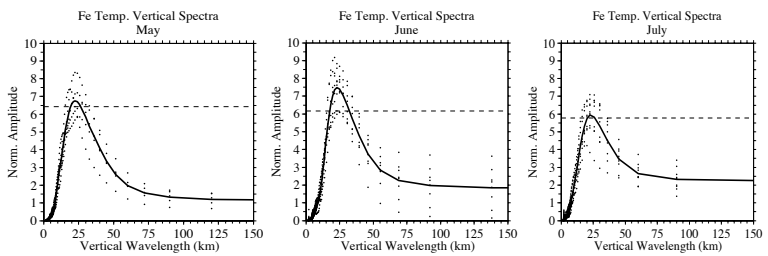

Figure 7. Mean vertical spatial spectra in the MLT region in May, June and July. Dotted lines are spectra for each episode.

\section{Amplitude}

We then plot the wave amplitude (standard deviation) with altitudes in Figure 8. The waves have been categorized into three groups: 3-5 h, 5-9 h, and 9-11 h. At least in May and June the 5-9 $\mathrm{h}$ wave amplitude clearly has a faster growth rate than the other two groups of waves, suggesting less damping encountered, or less saturated.
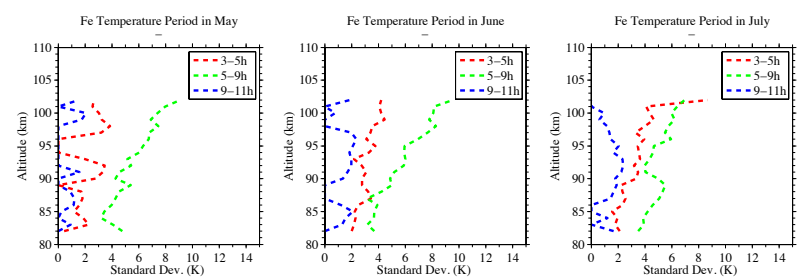

Figure 8. Standard deviations of the wave perturbations change with altitude for waves periods between 3-5 h, 5-9 h, 9-11 h in May, June, July.

\section{CONCLUSIONS AND DISCUSSION}

Large-amplitude ( $\pm 30 \mathrm{~K}$ ), long-period (4 to $9 \mathrm{~h}$ ) upward propagating waves are frequently observed in the MLT temperatures at McMurdo. Simultaneous observations of such waves using lidar, radar and airglow imager has revealed that one of the wave events on 11 June 2013 has the following properties: $\tau \sim 5 \mathrm{~h}, \lambda_{\mathrm{z}} \sim 20 \mathrm{~km}, \lambda_{h} \sim 1100 \mathrm{~km}$, propagating from north to south at azimuth $\theta \sim 180^{\circ}$.

Statistical study has shown that, unlike ordinary GWs, these waves have higher energy than the predicted universal power-law spectra, and very high occurrence frequencies. The discrete distribution of the dominant periods is also a distinct feature for these waves. Shortperiod ANM, 6-h or terdiurnal tides cannot be the cause of these waves because of the short vertical wavelength $\left(\lambda_{\mathrm{z}} \sim 22 \mathrm{~km}\right)$. Planetary scale Inertio-gravity waves simulated by Mayr et al. [10] at high latitudes have $\tau \sim 10$ $\mathrm{h}, \lambda_{\mathrm{z}} \sim 20 \mathrm{~km}$, is one possible candidate. These waves are related to the Class I gravity mode discussed in the classical literature. However, Mayr et al. only showed waves with $\tau \sim 10 \mathrm{~h}$. Whether their simulations also have periodicity between 4 and $9 \mathrm{~h}$ is unknown. The planetaryscale IGWs by Mayr et al. have zonal wave number 0-4, and long horizontal wavelength $\lambda_{h} \sim 4000 \mathrm{~km}$. Multiplestation observation network is required to examine the zonal structure of the 4-9 h waves.

\section{ACKNOWLEDGements}

We sincerely acknowledge Jeff Forbes and Chet Gardner for invaluable discussion. We appreciate the staff of United States Antarctic Program, McMurdo station, Antarctica New Zealand, and Scott Base for their superb support. This work was supported by NSF grants ANT0839091 and PLR-1246405. C.C. was partially supported by AGS-1136272.

\section{REFERENCES}

[1] Chu, X., Z. Yu, C. S. Gardner, C. Chen, and W. Fong, 2011: Lidar observations of neutral Fe layers and fast gravity waves in the thermosphere $(110-155 \mathrm{~km})$ at McMurdo $\left(77.8^{\circ} \mathrm{S}, 166.7^{\circ} \mathrm{E}\right)$, Antarctica, Geophys. Res. Lett., 38, L23807, doi:10.1029/2011GL050016.

[2] Chen, C., et al., 2013: Inertia-gravity waves in Antarctica: A case study using simultaneous lidar and radar measurements at McMurdo/Scott Base, $J$. Geophys. Res., 118, doi:10.1002/jgrd.50318.

[3] Forbes, J. et al., 1999: Lamb waves in the lower thermosphere: Observational evidence and global consequences, J. Geophys. Res., 104, doi:

10.1029/1999JA900044

[4] Mosegaard, K., \& Tarantola, A., 1995: Monte Carlo sampling of solutions to inverse problems. J. Geophys. Res., 100, doi:10.1029/94JB03097

[5] Nakamura, T. et al., 1993: Characteristics of gravity waves in the mesosphere observed with the middle and upper atmosphere radar 2. Propagation direction, $J$. Geophys. Res., 98, doi:10.1029/92JD03030.

[6] Chen, C. et al., 2012: Lidar and radar investigation of inertia gravity wave intrinsic properties at McMurdo, Antarctica, Proceedings of the 26th ILRC, Greece.

[7] Gardner, C. S., 1994: Diffusive filtering theory of gravity wave spectra in the atmosphere, J. Geophys. Res., 99, doi:10.1029/94JD00819.

[8] Lu et al., 2015: Vertical evolution of potential energy density and vertical wave number spectrum of Antarctic gravity waves from 35 to $105 \mathrm{~km}$ at McMurdo $\left(77.8^{\circ} \mathrm{S}\right.$, $\left.166.7^{\circ} \mathrm{E}\right)$, J. Geophys. Res., accepted.

[9] Zink, F., \& Vincent, R. A., 2001: Wavelet analysis of stratospheric gravity wave packets over Macquarie Island: 1. Wave parameters, J. Geophys. Res., 106, doi: 10.1029/2000JD900847.

[10] Mayr, H. G. et al., 2004: Properties of internal planetary-scale inertio gravity waves in the mesosphere. Ann. Geophys., 22, doi:10.5194/angeo-22-3421-2004. 